\title{
SÉRIE HISTÓRICA DE CUSTOS COM TERAPIA DE SUBSTITUIÇÃO RENAL NO MUNICÍPIO DO RIO DE J ANEIRO (1995-2009)
}

Time series of costs of renal replacement therapy in Rio de J aneiro city (1995-2009)

Serie histórica de costes con terapia de reemplazo renal en la ciudad de Rio de J aneiro (1995-2009)

\section{RESUMO}

Objetivou-se avaliar a tendência temporal do custo total de terapia de substituição renal e a proporção entre custo e solicitações de Autorizações de Procedimentos Ambulatoriais de Alta Complexidade/Custo no município do Rio de Janeiro, entre 1995 e 2009, em totais e segundo prestador. Estudo quantitativo, descritivo, tipo série temporal. Utilizaram-se informações referentes aos valores e quantidades de autorizações de procedimentos de alta complexidade para terapia de substituição renal por ano, disponíveis no DATASUS, coletadas entre fevereiro e março de 2011. Observou-se tendência crescente para o custo total e o total de autorizações destes procedimentos ( $y=3,8414 x+16,904, R^{2}=0,9665$ e $y=14519 x+299719, R^{2}=0,8835$, respectivamente). Há uma diferença estatisticamente significativa $(p<0,001)$ da variação da tendência quando comparados os serviços públicos e os privados. Conclui-se que há uma tendência em programar cada vez mais serviços nos hospitais públicos, tornando crescente a demanda por profissionais qualificados para atuação na área.

Palavras-chave: Epidemiologia. Terapia de substituição renal. Planejamento em saúde.

\begin{abstract}
The study objective was to evaluate the temporal tendency of the final cost of renal replacement therapy and also to evaluate the ratio between a procedure cost and its requests on the High Complexity Outpatient Procedures Authorization/Cost in the city of Rio de Janeiro, between 1995 and 2009 in totals and as the second provider. In this quantity, grade, descriptive and retrospective study, we used information about the values and quantities of high-complexity procedures licenses for renal replacement therapy each year, available on DATASUS, collected between February and March of 2011. An increasing trend was observed for the total cost and authorizations for high-complexity procedures for RRT ( $y=3.8414 x+16.904, R^{2}=0.9665$ and $y=14519 x+299719, R^{2}=0.8835$, respectively). There is a statistically significant difference $(p<0.001)$ in the range of trend, when comparing public and private institutions. The main conclusion is that there is a tendency to book a large number of services and procedures in public hospitals, which increases the need of qualified professionals to work in the area.
\end{abstract}

Keywords: Epidemiology. Renal replacement therapy. Health planning

\section{Resumen}

El objetivo del estudio fue evaluar la tendencia temporal del coste total de terapia de reemplazo renal y la proporción entre coste y solicitaciones de Autorizaciones de Procedimientos en Ambulatorio de Alta Complejidad/Coste en la cuidad de Rio de Janeiro, entre 1995 y 2009, en total y segundo proveedor. Estudio cuantitativo, descriptivo, de serie temporal. Se utilizó la información relativa a los valores y las cantidades de los procedimientos de permisos de alta complejidad para la terapia de reemplazo renal por año, disponible en DATASUS, recolectadas desde febrero y marzo de 2011. Se observó una tendencia creciente para el coste total y el total de permisos de estos procedimientos $\left(y=3,8414 x+16,904, R^{2}=0,9665\right.$ e $y=$ $14519 x+299719, R^{2}=0,8835$, respectivamente). Hay una diferencia estadísticamente significativa $(p<0,001)$ de la variación de la tendencia al comparar pública y privada. Se concluye que hay una tendencia a establecer cada vez más servicios en los hospitales públicos, por lo que la creciente demanda de profesionales cualificados para trabajar en el área.

Palabras clave: Epidemiología. Terapia de reemplazo renal. Planificación en salud. 


\section{INTRODUÇÃO}

A Terapia de Substituição Renal (TSR) tem por finalidade suprir de maneira parcial ou integral a função dos rins e, além disso, corrigir o metabolismo orgânico. É empregada em indivíduos que apresentam Injúria Renal Aguda (IRA), Doença Renal Crônica (DRC), além de englobar o transplante renal ${ }^{1-2}$. Assim, o Ministério da Saúde custeia tanto a cobertura do procedimento quanto o fornecimento de medicações excepcionais aos indivíduos em TSR por meio da Autorização de Procedimentos Ambulatoriais de Alta Complexidade/Custo $(\mathrm{APAC})^{3}$.

No Brasil, somente no ano de 2004, o gasto com TSR ultrapassou $R \$ 1$ bilhão. Em 2009, a taxa de prevalência de terapia dialítica foi de 405 por milhão da população (pmp) tendo como doenças de base principais a hipertensão arterial (35\%) e o diabetes melito (27\%), o total de pacientes em diálise no país foi de 77.589 pessoas, sendo o Sistema Único de Saúde responsável por $86,9 \%$ dos pagamentos referentes ao custo da TSR neste mesmo $\mathrm{ano}^{4-5}$.

Ainda em 2009, a porcentagem dos pacientes em hemodiálise (HD) foi 86,9\% e o número de sessões pagas passou de 10 milhões, gerando gasto de 1,5 bilhão de reais ${ }^{2,4,6}$. No município do Rio de Janeiro, somente no primeiro trimestre de 2012, o custo com TSR, modalidade HD, ultrapassou 20 milhões de reais $(R \$ 20.468 .140,40)^{7}$. Sendo assim, o debate referente ao gasto com TSR e seu impacto no orçamento do governo chama a atenção pela expressividade por ele representada.

Estudos sobre custo com TSR ainda são incipientes, sendo encontrados resultados de pesquisas englobando apenas uma ou outra modalidade terapêutica, em geral a HD, por ser a modalidade mais abrangente. Nos estudos encontrados, o valor médio gasto por paciente/ano foi 21.595 euros na Espanha ${ }^{8}$, 89.154 dólares canadenses no Canadá9 $\mathrm{e}$ 28.033,71 euros em Portugal ${ }^{10}$. No Brasil, em 2012, o valor pago pelo Ministério da Saúde foi alterado de $R \$ 155,00$ para $R \$ 170,50$ enquanto na Espanha o valor da sessão de HD é 129,96 euros ${ }^{8,11}$. 0 custo das sessões de HD tem sido ponto de discussão constante entre as entidades representantes dos centros de diálise e da especialidade e órgãos do governo.

A partir deste contexto, temos como objetivo avaliar a tendência temporal do custo total de terapia renal substitutiva e a proporção entre custo e solicitações de Autorizações de Procedimentos de Alta Complexidade no município do Rio de Janeiro, entre 1995 e 2009, em totais e segundo prestador.

A discussão do custo da TSR e sua relação com o valor pago pelo SUS merece investigação haja vista a lacuna existente nas publicações nacionais acerca do seu custo e a distribuição por prestador. Assim, a contribuição desse estudo está na possibilidade de discutir do impacto no orçamento gerado pala TSR, o que pode se configurar uma ferramenta a ser utilizada na gestão dos serviços que oferecem o tratamento auxiliando no planejamento da cobertura, na distribuição dos recursos financeiros e materiais, além de recursos humanos especializados para atender à demanda crescente, como assinala a Lei Orgânica da Saúde em diversos dos seus artigos.

\section{MÉTODO}

A articulação entre a epidemiologia e o planejamento de saúde é um dos temas de maior destaque entre pesquisadores, docentes e profissionais dos serviços nos últimos anos. Esta discussão ocorre tanto a partir da necessidade de retroalimentação dos estudos epidemiológicos pela realidade local quanto pela tentativa de tornar a epidemiologia e, por conseguinte, a vigilância epidemiológica, uma ferramenta de maior qualidade técnica.

De modo geral, os aspectos abordados referem-se ao papel da epidemiologia na definição do objeto do planejamento de saúde, em vários níveis de gestão do sistema. Isso se define na utilização do chamado "enfoque epidemiológico" no processo de formulação de políticas, na definição de critérios para repartição de recursos, na elaboração de diagnósticos e análises de situações de saúde, na elaboração de planos e programas, bem como na organização de ações e serviços e avaliação de sistemas, políticas, programas e serviços de saúde em geral. Frutos desta discussão estão os Planos Diretores para 0 Desenvolvimento da Epidemiologia, cuja versão mais recente é o IV Plano Diretor ${ }^{12}$.

Nessa perspectiva, vários avanços metodológicos e instrumentais vêm sendo propostos e aperfeiçoados, notadamente no que se refere aos Sistemas de Informação em Saúde, aos processos de análise da situação e ao planejamento de ações da vigilância em saúde. A ideia é de que a análise de situação de saúde, com o suporte da epidemiologia, pode se colocar como possibilidade de construção de uma nova prática sanitária entendida como uma forma de organização e operacionalização do sistema que enfatize as ações intersetoriais de promoção da saúde e as ações e serviços de prevenção de riscos e agravos junto a grupos populacionais priorizados. Considera-se que a reorientação do planejamento e, em última análise, da gestão, do financiamento, da organização do modelo assistencial do sistema são processos que não podem prescindir da epidemiologia, como prática instrumental ${ }^{12}$.

Este estudo do tipo descritivo de série temporal avaliou o custo total de TSR e a proporção entre total de solicitações de Autorizações de Procedimentos de Alta Complexidade (APAC) no Município do Rio de Janeiro, entre 1995 e 2009. Foi avaliado ainda o total de custo segundo prestador de serviço (federal, estadual, municipal e privado - com e sem fins lucrativos). Os dados utilizados neste estudo são oriundos da base de dados secundários das APAC do SIA-SUS, e foram obtidos na página oficial do 
Departamento de Informática do SUS (DATASUS) ${ }^{6} \mathrm{em}$ coleta realizada nos meses de fevereiro e março de 2011. As variáveis eleitas para obtenção dos dados foram referentes aos valores de APAC por ano, bem como à proporção entre o número de solicitações de APAC emitidas e as suas aprovações, criando um indicador nomeado "proporção de autorizações".

As informações foram ainda desagregadas segundo tipo de prestador, sendo públicas as instituições federais, estaduais e municipais; e as privadas, com ou sem fins lucrativos. $\mathrm{Na}$ análise das tendências de regressão foi utilizado o modelo polinomial, no qual a proporção de custos, proporção de procedimentos, gasto total e número de autorizações foram consideradas como variáveis dependentes $(Y)$ e os anos calendário de estudo, como variáveis independentes (X). Para evitar a autocorrelação entre os termos da equação de regressão, foi feita a transformação da variável ano na variável ano-centralizada ( $X$ menos o ponto médio da série histórica).

Inicialmente, foram construídos diagramas de dispersão entre os indicadores e os anos de estudo para visualizar a função que poderia expressar a relação das tendências dos indicadores e escolher a ordem do polinômio para análise. Ainda, para avaliar a diferença na tendência dos custos por tipo de prestador, foi realizado o teste de Kruskal-Wallis. Foi considerada tendência significativa aquela cujo modelo estimado obteve $p<0,05$. Foi feita a análise de resíduos e, para todos os modelos, observaram-se a suposição de homocedasticidade e a aderência à distribuição normal ${ }^{13}$. Para análises das tendências foi utilizado o programa SPSS (versão 19.0).

\section{RESULTADOS}

Os gráficos 1 e 2 apresentam, respectivamente, a tendência temporal para os valores gastos em TRS e a quantidade de procedimentos autorizados no município do Rio de Janeiro, entre 1995 e 2009. Observou-se que a tendência é crescente para o custo total e o total de autorizações de APAC para terapia renal substitutiva $(\mathrm{y}=$ $3,8414 x+16,904, R^{2}=0,9665$ e $y=14519 x+$ $299719, R^{2}=0,8835$, respectivamente), evidenciando um aumento de gasto de cerca de $48 \%$ ao longo de 15 anos.

Além disso, cabe ressaltar que há uma variação crescente, porém mais estável da proporção entre terapias solicitadas e autorizadas, tanto em valores quanto em número $\left(y=0,0006 x+0,9926, R^{2}=0,3356\right.$ e $y=$ $0,0007 x+0,9924, R^{2}=0,3702$, respectivamente), o que mostra relativa organização da rede de assistência em nefrologia no Município, de forma a assegurar a cober tura do serviço de terapia complementar.

Gráfico 1 - Estatísticas dos valores de Autorização para Procedimentos de Alta Complexidade para Terapia de Substituição Renal. Rio de Janeiro, 1995-2009.

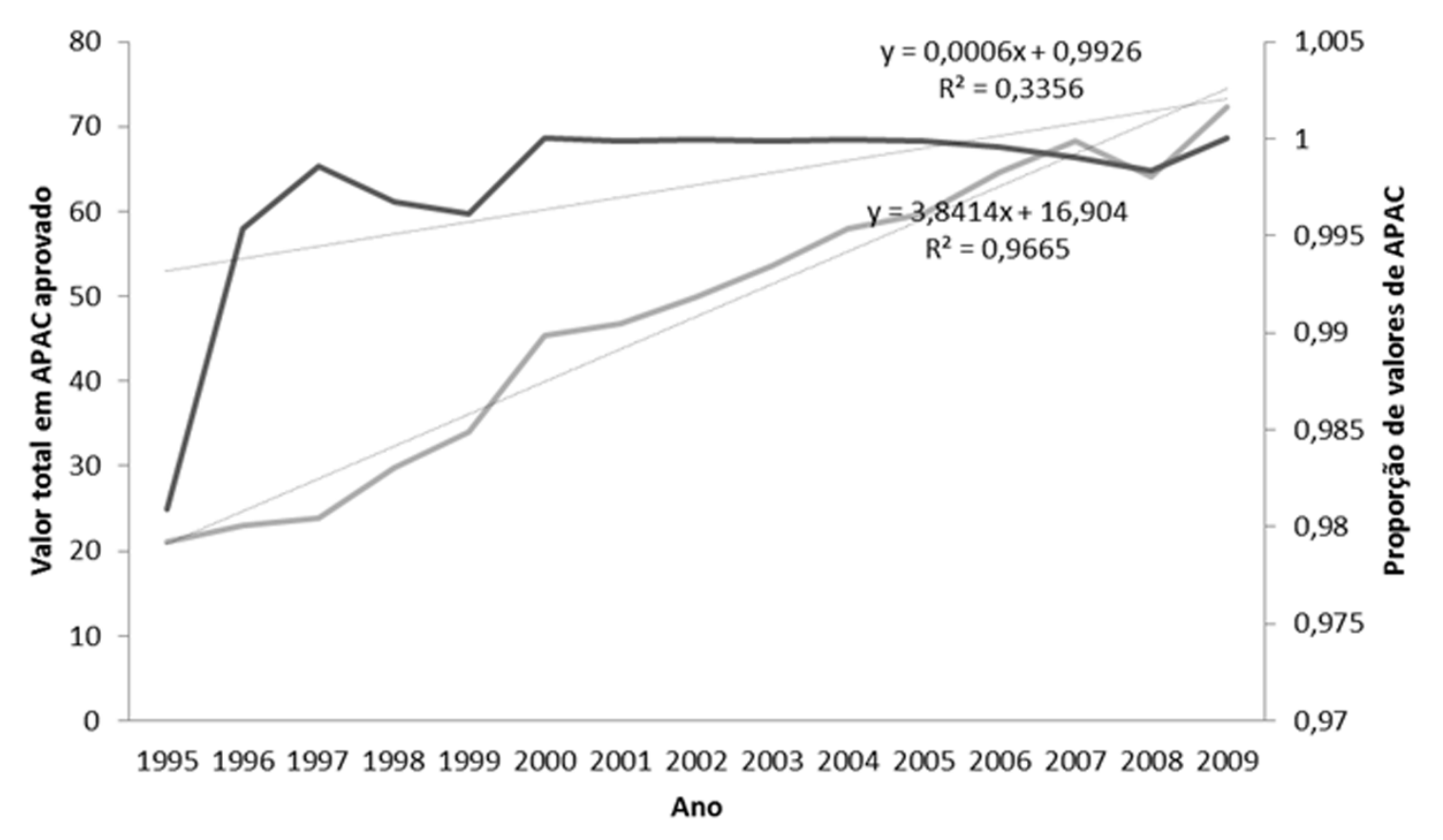


Gráfico 2 - Estatísticas de Autorização para Procedimentos de Alta Complexidade para Terapia de Substituição Renal. Rio de Janeiro, 1995-2009.

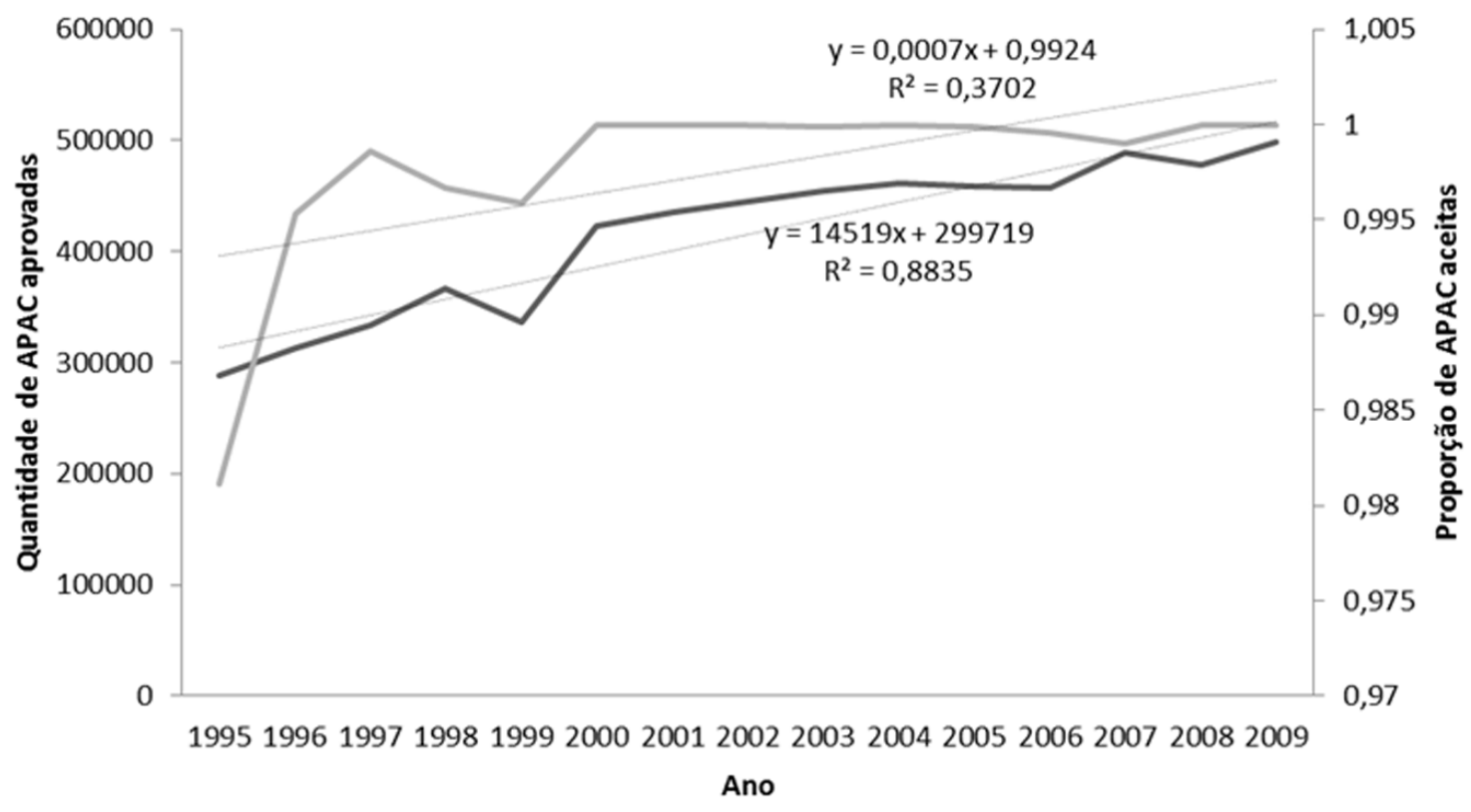

Avaliou-se, ainda, que há uma diferença fins lucrativos), com nítido aumento da oferta pública e estatisticamente significativa $(p<0,001)$ da variação da redução da privada, evidenciando o incremento na gestão tendência quando comparados os serviços públicos da oferta desta linha de cuidado por parte do SUS (Tabela (federais, estaduais e municipais) e os privados (com e sem 1).

Tabela 1 - Custo de TSR segundo tipo de prestador. Rio de Janeiro, 1995-2007*.

\begin{tabular}{lllllll}
\hline \multirow{2}{*}{ Ano } & \multicolumn{7}{c}{ Tipo de Prestador } & P valor** \\
\cline { 2 - 6 } & Federal & Estadual & Municipal & $\begin{array}{c}\text { Privado com fins } \\
\text { lucrativos }\end{array}$ & $\begin{array}{c}\text { Privado sem fins } \\
\text { lucrativos }\end{array}$ & Total \\
\hline 1995 & 1447234 & 5138230 & 12349889 & 3909468 & 4889342 & 34165837 \\
1996 & 1313289 & 8139975 & 17632549 & 1899890 & 3920478 & 38494515 \\
1997 & 1272742 & 6413216 & 18670185 & 3846065 & 3485983 & 38907927 \\
1998 & 1226390 & 8727658 & 17186150 & 1826776 & 2472226 & 36439703 \\
1999 & 1164846 & 8926947 & 23644738 & 1831831 & 2447787 & 43017945 \\
2000 & 1264024 & 12512583 & 27455470 & 1769127 & 2398872 & 50312510 \\
2001 & 1105605 & 14449879 & 24543894 & 1455257 & 2731378 & $48994864<0,001$ \\
2002 & 1129281 & 13849549 & 22799322 & 1490666 & 2700710 & 46652616 \\
2003 & 2543141 & 13559974 & 20823974 & 1392137 & 2180035 & 43170935 \\
2004 & 3464764 & 15910997 & 18807110 & 1331019 & 2021249 & 43782627 \\
2005 & 3444169 & 20061145 & 18109162 & 1370761 & 2249870 & 47483094 \\
2006 & 3574696 & 21240573 & 19035929 & 1555139 & 2057519 & 49495581 \\
\hline 2007 & 4715700 & 21419640 & 19964191 & 1356585 & 1276750 & 50585482 \\
\hline
\end{tabular}

*Avaliado para o período mencionado por mudanças das formas de pagamento a partir de 2008, inviabilizando a comparação com o período em questão.

** Obtido por meio do teste de Kruskal-Wallis

Fonte: DATASUS, 2011. 


\section{DISCUSSÃO}

Dos indivíduos que necessitam de TSR, a DRC mostra-se como a maior causa, tendo como principais doenças de base, respectivamente a HAS e o DM. No período de 2000 a 2010, o número de indivíduos sob TSR no país aumentou mais de 100\%, passando respectivamente de 42.695 para 92.0914-5,7. Na região sudeste, o crescimento de indivíduos sob TSR tem aumentado nos últimos anos, o que também pode ser notado no município do Rio de Janeiro. Assim, conhecer a oferta, utilização e gastos dos recursos é essencial, pois fornece informações relevantes ao subsídio de decisões que promovam maior equidade ${ }^{14}$.

Ainda sobre isso, tem-se a ideia de que a análise de situação de saúde, com o suporte da epidemiologia, pode se colocar como possibilidade de construção de uma nova prática sanitária entendida como uma forma de organização e operacionalização do sistema que enfatize as ações inter-setoriais de promoção da saúde e as ações e serviços de prevenção de riscos e agravos junto a grupos populacionais priorizados. Considera-se que a reorientação do planejamento e, em última análise, da gestão, do financiamento, da organização do modelo assistencial do sistema são processos que não podem prescindir da epidemiologia, como prática instrumental|13-14.

Na questão em destaque, ou seja, para a discussão sobre a atenção às TSR percebe-se a ampliação da oferta deste serviço por parte do serviço público, o que assinala para a questão da demanda-oferta haja vista que o aumento do serviço especializado, sem crescimento de profissionais especializados, como enfermeiros e demais especialistas em nefrologia atuando clinicamente, pode reproduzir impactos significativos no que tange ao custo e/ou qualidade do serviço ofertado. Ainda sobre esta questão é importante enfatizar que a especialização deve ser um fator a ser considerado nos concursos públicos para provimento de vagas que atendam a esta demanda ${ }^{15}$.

É válido ressaltar que o Brasil tem o quarto maior número de pacientes mantidos em diálise ${ }^{16}$, sem considerar 0 envelhecimento da população, aumento da expectativa de vida e 0 aumento da prevalência das doenças crônicas, dentre elas a doença renal.

Outras características devem ser levadas em consideração no planejamento à saúde, principalmente tratando-se da oferta de serviços especializados no Brasil; um exemplo seria a grande desinformação de pacientes e familiares assistidos sobre seu real problema de saúde. Geralmente são pacientes acometidos por uma doença de base que compromete o sistema renal, refletindo em impacto direto na autonomia do indivíduo $0^{10-17}$.

No Brasil, são variados os tipos de ações e serviços de saúde, podendo ser públicos, privados, com ou sem fins lucrativos, além daqueles operados por operadoras de planos de saúde, 0 que dificulta medir, de maneira geral, a utilização dos serviços ${ }^{14}$. Os dados evidenciaram um aumento pela busca dos serviços públicos em detrimento dos serviços privados, que garantem não só as sessões de hemodiálise, mas também a oferta de medicação e internação hospitalar, conforme disposto pelo Ministério da Saúde?.

Nesse sentido, em resposta às várias demandas junto ao Ministério da Saúde para a revisão do modelo de prestação de serviços de TSR do SUS, instituiu-se um Grupo de Trabalho com o objetivo de realizar um diagnóstico sobre a situação da doença renal no país. Ampliando o escopo do problema, o Ministério da Saúde resolveu elaborar a Política de Atenção aos Portadores de Doenças Renais, datada de $2004^{18}$.

Tal decisão revela a disposição política do Ministério da Saúde de mudar o modelo existente que é baseado em procedimentos, centrado no alto custo, caracterizado pela ausência de gestão pública e de estratégias de integração entre os diversos níveis de atenção. Isso impõe a criação de um modelo de atenção e gestão em que os pacientes sejam abordados de forma integral e integrada, com medidas de curto, médio e longo prazo, buscando modificar de forma positiva o perfil epidemiológico da doença renal no Brasii ${ }^{18}$.

No SUS, cerca de $30 \%$ de todo o orçamento é gasto com procedimentos de alta complexidade, atendendo apenas a $3 \%$ dos usuários do sistema. Por conseguinte, a alta complexidade é frequentemente responsabilizada pelos custos elevados despendidos em saúde. Desta feita, a discussão precisa ser ampliada, portanto, questionando se todo 0 "investimento em saúde" tem reflexo coletivo. Ou seja, avaliar qual é o impacto epidemiológico da autorização de procedimentos de alta complexidade sem levar em conta protocolos que atendam ao princípio da integralidade do SUS.

Em seus aspectos mais importantes, a Política instituída em 2004 passou a prever a integralidade no cuidado das doenças crônicas não transmissíveis, com a introdução das linhas de cuidado integral, incluindo prevenção, promoção, tratamento e reabilitação. A proposta da linha de cuidado supõe que o usuário do sistema tenha um acompanhamento contínuo, como resultado da articulação dos vários níveis de atenção à saúde.

\section{CONCLUSÕES}

É notório o aumento da demanda por serviços de TSR no município do Rio de Janeiro, em termos de quantidade de serviços e valores por eles consumidos, e a ampliação do acesso a este tipo de terapia complementar, em especial nos serviços públicos, revela a expansão da cobertura de TSR pelo SUS sem precisar realizar contratação de serviços particulares, sejam eles com ou sem fins lucrativos. Tal fato mostra uma tendência em programar cada vez mais serviços nos hospitais públicos, tornando crescente a demanda por profissionais qualificados para atuação na área.

Observa-se que, no campo da nefrologia, é exigido dos profissionais especializados, especialmente do enfermeiro, cada vez mais um incremento na qualidade da assistência, 
principalmente por ser este o profissional que maior tempo se manterá próximo ao paciente. Com vistas a atender este objetivo, deve-se investir em qualificação no que tange aspectos técnicos e científicos do profissional que trabalha nesta área.

Contudo, a literatura escassa, no que diz respeito ao custo da TSR, relacionando o gasto total e o tipo de prestador, trouxe para o estudo a limitação na discussão aprofundada da variação da tendência do custo nos diferentes tipos de serviço, sobretudo pela dificuldade de se obterem dados referentes ao pagamento das APACs a serviços privados, embora estes constituam a minoria da cobertura dos serviços de TSR.

Aponta-se a necessidade de ampliar estudos sobre a temática a fim de que os dados produzidos possam nortear as aç̃̃es programáticas de distribuição de recursos e cobertura dos serviços de TSR no município, atendendo a clientela que cresce exponencialmente.

\section{REFERÊNCIAS}

1. Lima EX. Assistência de Enfermagem na Insuficiência Renal Aguda. In: Lima EX, Santos I, Souza ERM. Tecnologia e o cuidar de enfermagem em terapias renais substitutivas. São Paulo: Atheneu; 2009.

2. Mehta RL, Kellum JA, Shah SV, et al. Acute Kidney Injury Network: report of an initiative to improve outcomes in acute kidney injury. Crit Care. 2007; 11(2): R31.

3. Portaria n. 205, de 6 de novembro de 1996. Implanta formulários/ instrumentos e regulamentar suas utilizações na sistemática de autorização e cobrança dos procedimentos ambulatoriais de alta complexidade/custo e fornecimento de medicamentos excepcionais a pacientes em Terapia Renal Substutiva - T.R.S. Diário Oficial da República Federativa do Brasil, Brasília(DF), (08/11/1996): Seção I

4. Sesso RCC, Lopes AA, Thomé FS, Lugon JR, Burdmann EA. Censo brasileiro de diálise, 2009. J. bras. nefrol. 2010; 32(4): 380-4.

5. Salgado Filho N, Brito DJA. DRC: a grande epidemia deste milênio. J. bras. nefrol 2006 set.; 28(3 supl.2): 1-5.

6. DATASUS. Procedimentos hospitalares no SUS [citado em 2011 abr. 1]. Disponível em <http://tabnet.datasus.gov.br $>$.

7. Associação Brasileira dos Centros de Diálise e Transplante. TSR; 2012. Disponível em: <http://www.abcdt.org.br>.

8. Barreiro JML, Suarez MA, Alonso JAS, Martínez AG. Costes y valor añadido de los conciertos de hemodiálises y diálisis peritoneal. Nefrología(Madri). 2001; 31(6): 656-63.

9. McFarlane P, Komenda P. Economic considerations in frequente home hemodialysis. Semin Dial; 2011 nov./dec.; 24(6): 678-83.
10. Rocha MJ, Ferreira S, Martins LS, Almeida M, Dias L, Pedroso $S$, et al. Cost analysis of renal replacement therapy by transplant in a system of bundled payment of dialysis. Clin Transplant. 2011 jul./aug.; 25(6): 821-9.

11. Portaria n. 165, de 7 de março de 2012. Altera os valores de remuneração dos procedimentos de Terapia Renal Substitutiva abaixo descritos, constantes do grupo 3 da Tabela de Procedimentos, Medicamentos, Órteses, Próteses e Materiais Especiais do Sistema Único deSaúde - SUS. Diário Oficial da República Federativa do Brasil, Brasilia(DF), (08/03/2012): Seção 1

12. Gordis L. Epidemiology and Health Policy. In: Gordis, L. Epidemiology. 3. ed. Porto Alegre: Artmed; 2008. p.333-347.

13. Rothman K, Greenland S, Lash T. Epidemiologia moderna. 3. ed. Porto Alegre(RS): Artmed; 2008.

14. Kajiura AP. Equidade e alocação de recursos no Sistema Único de Saúde: análise de procedimentos em otorrinolaringologia. [dissertação] Recife (PE): Fundação Oswaldo Cruz; 2010.

15. Silva RC, Ferreira MA. Características dos enfermeiros de uma unidade tecnológica: implicaç̃̃es para o cuidado de enfermagem. REBEN. 2011 jan./fev; 64(1): 98-105.

16. Sancho LGe Dain S. Análise de custo-efetividade em relação às terapias renais substitutivas: como pensar estudos em relação a essas intervenções no Brasil? Cad. saúde pública. 2008 jun.; 24(6): 1279-90.

17. Santos I, Rocha RPF, Berardinelli LMM. Qualidade de vida de clientes em hemodiálise e necessidades de orientação de enfermagem para 0 autocuidado. Esc. Anna Nery Rev. Enferm. 2011 jan./mar,; 15(1): 31-8.

18. Portaria n. ${ }^{0}$ 1168/GM, de 15 de junho de 2004. Institui a Política Nacional de Atenção ao Portador de Doença Renal, a ser implantada em todas as unidades federadas, respeitadas as competências das três esferas de gestão. Diário Oficial da República Federativa do Brasil, Brasília(DF): 17/06/2004, Seção 1 